difference between two colours, uniform chromaticity scales, colour difference formulae and colour tolerances. 'The chapter on practical applications has been extended, particularly in the section on dyeing. This now deals with colorant formulation methods based on KubelkaMunk theory made possible by modern computers. Smaller additions and revisions have been made to other parts of the book and the references; the appendices have been enlarged to include tables of the energy distribution of illuminants $D_{5500}, D_{6500}$ and $D_{7500}$ and the $196410^{\circ}$ distribution coefficients weighted by energy values of these illuminants.

It is difficult to find anything but praise for this book for content and printing; revision of units throughout the book could have extended to the use of SI units for luminance on page 42; and the colour patches on the chromaticity diagram of Plate 7 have been changed from circles to trapezia but the legend still refers to circles.

A number of books on colour science and technology of various levels of complexity have appeared in recent years, but there can be no doubt that this book will still hold its place as an excellent introduction to the subject and can be recommended unreservedly.

\section{A. Humphreys}

\section{HILBERT SPACES}

\section{Introduction to Spectral Theory in Hilbert Space}

By Gilbert Helmberg. (North-Holland Series in Applied Mathematics and Mechanics, Vol. 6.) Pp. xiii +346. (North-Holland: Amsterdam and London, 1969.) $140 s$.

THE contents of this book can best be described by asking in this review some of the questions posed by the author himself in the preface to his book, such as, what is a Hilbert space, what is a bounded operator in a Hilbcrt space, what is a (not necessarily bounded) self-adjoint operator in Hilbert space, and so on. To all these questions the reader will find full and adequate answers in the text of the book.

Chapters one and two are concerned with the concept and elementary properties of Hilbert spaces including the geometry of such spaces. The spaces $l_{2}$ (summable-square sequences) and $L_{2}$ (integrable-square functions) are considered in some detail and used to illustrate many of the properties developed for general Hilbert spaces. Chapter three is devoted to the study of bounded linear operators and develops the theory of adjoint, self-adjoint, normal and projection operators. There is a detailed discussion of the Fourier-Plancherel operator to illustrate several of the concepts introduced in this chapter. In chapter four, the general theory of linear operators in Hilbert spaces is developed and includes the theory of unbounded operators and their adjoints, the concept of a closed operator, the idea of invariant subspaces of an operator and a careful account of the spectrum of an operator with special reference to self-adjoint and unitary operators. Examples given are the differentiation and multiplication operators in the $L_{2}$ spaces, and the Fourier-Plancherel theory is used to link the properties of those two operators. Chapter five contains a standard acccunt of the spectral properties of compact operators with applications to the theory of Fredholm operators. The spectral analysis of bounded linear operators is presented in chapter six, which considers in detail the cases of self-adjoint, unitary and normal operators; in all three cases the spectral theorem is proved in detail. The final chapter, seven, applies the ideas of previous chapters to develop the spectral theorem for unbounded self-adjoint operators; examples are discussed, in particular the differentiation operator of previous chapters.

There are two appendices; the first is on the graph of a linear operator, while the second contains a brief account of the properties of the Riemann-Stieltjes and Lebesgue integrals needed elsewhere in the book. There is a detailed bibliography, an index of symbols and a subject index. The book has been clearly and carefully printed.

I can recommend this book as a most helpful introduction to spectral theory in Hilbert space. The author has clearly taken great care in the preparation of his text and has produced a book which should be of considerable help to students and research workers in this subject. The price is high, but the serious reader will find, nevertheless, that this book will be a valuable acquisition for his library shelves.

W. N. EveritT

\section{CURVE SYSTEMS}

\section{Systems of Frequency Curves}

By William Palin Elderton and Norman Lloyd Johnson. Pp. 216. (Cambridge University Press: London, June 1969.) $60 s ; \$ 10$.

Sir William Elderton's Frequency Curves and Correlation was originally published in 1906 . The present book is a fairly drastic revision of the fourth edition of this work. Most of the material on correlation is now removed. Also, whereas Elderton's discussion was largely limited to curves of the Pearson system, Professor Johnson has now extended the treatment to other systems including some curves with which his own name has now become particularly associated. There is also an interesting section on bivariate frequency surfaces.

The fitting of theoretical frequency curves to observed distributions of individual measurements played a more central part in statistical methodology early in the century than it does today. Its importance was undoubtedly overemphasized and in time "curve-fitting" as a statistical activity was very much called in question. Strangely, along with this criticism, there still continued much lively discussion of the problem of determining the best method of estimating the parameters of the fitted curves. Here, however, curve-fitting procedures were possibly being used to illustrate currently developing theories of statistical inference, rather than because of the intrinsic importance of the curves actually fitted to individual sets of data. Much talking at cross-purposes may have resulted from this. Johnson touches on some of these issues in an appendix but does not enter very far into discussion of them.

It was not only the graduation of distributions of individual observations, however, that gave impetus to the Pearson system. Very early, Pearson recognized the importance of special curves of the system in graduating the probability distributions of different sampling criteria. One may instance here the early development of the $x^{2}$ distribution. Later landmarks were the small-sample theory of Student and of R. A. Fisher. The competing Gram-Charlier and Edgeworth systems of curves were also found to have application to some of the more complex sampling problems not subsumed under the general linear hypothesis of normal theory. Although these systems are of an approximate nature, being based on series develop. ments, they have made possible some progress in the development of the small sample theory of otherwise intractable sampling problems. These various considerations may explain why Elderton's book has had continuing success for so long. The reader may not now go to this book for the reasons that the original author intended. But it does provide a systematic account of an area of statistical methodology which is lacking in most modern texts, but which still has relevance to many of the problems which statisticians today take up and discuss. Johnson has admirably preserved the spirit of the original work, while his additional material contributes considerably to its usefulness and should ensure for it a further long lease of life.
B. L. WFLCH 